\title{
Gridrec-MS: an algorithm for multi-slice tomography
}

\author{
Esther H. R. Tsal, ${ }^{1,2, *}$ (iD Federica Marone, ${ }^{1}$ (i)
${ }^{1}$ Paul Scherrer Institut, 5232 Villigen PSI, Switzerland \\ ${ }^{2}$ Brookhaven National Laboratory, Upton, New York 11973, USA \\ ${ }^{3} e$-mail: manuel.guizar-sicairos@psi.ch \\ *Corresponding author: etsai@bnl.gov
}

and Manuel Guizar-Sicairos ${ }^{1,3}$ (i)

Received 14 January 2019; revised 28 February 2019; accepted 13 March 2019; posted 18 March 2019 (Doc. ID 357549);

published 19 April 2019

\begin{abstract}
Advances in imaging systems and modeling allow for depth information to be retrieved from projections via virtual sectioning of the imaged object. Here we introduce a regridding method that explicitly and directly incorporates this information into a general and non-iterative tomographic reconstruction algorithm. The method is applicable to any imaging scheme that provides depth-resolved projections. Additionally, we show, via numerical simulations, that with this method the required number of projections for adequate angular sampling can be reduced. (c) 2019 Optical Society of America
\end{abstract}

https://doi.org/10.1364/OL.44.002181

Provided under the terms of the OSA Open Access Publishing Agreement

One general limitation of an imaging system is the depth of field (DOF), which refers to the distance in the beam propagation direction $z$ within which an imaged object remains in focus. The DOF leads to a limit on either the sample thickness or the overall imaging resolution. Various methods have been developed to extend the DOF, e.g., $[1,2]$. On the other hand, the limited DOF can be exploited to directly obtain sectioning or 3D information about the specimen. Recent advances in imaging techniques allow for extracting some depth information from a projection through signal processing or physical modelling [1,3-7]. For example, the multi-slice method is applied in coherent diffraction imaging to extend the DOF [8-10]. For a tomographic system with limited DOF, the depth information can in principle be extracted by the aforementioned methods and subsequently incorporated into the tomographic reconstruction to improve the resolution or reduce the angular sampling requirement. It has been shown that better depth resolution can be obtained by slightly rotating the sample at limited angles $[11,12]$. The reverse has also been demonstrated, i.e., the angular sampling for tomography can be reduced given depth-resolved projections [13-16]. However, so far little has been done for designing general reconstruction methods that incorporate such depth information directly and effectively. Tomographic reconstructions typically assemble projections with sufficient angular sampling to deliver a 3D tomogram with isotropic resolution through, for example, filtered back projection [17], algebraic reconstruction [18], or regridding methods [19]. As a trivial solution, multiple sections at different depths can be simply combined to give conventional projections for tomography, however in this manner the depth resolution information is lost and the Crowther's criterion cannot be relaxed [15]. Some multi-slice tomographic reconstruction approaches $[14,16,20]$ are intrinsically limited to ptychography. In optical microscopy, multi-slice tomography has been demonstrated from multiple-view data, either from focus series $[21,22]$ or by fluorescence with illumination sectioning [23]. 3D reconstructions are performed by image rotation followed by a step of image fusion, in which the multi-slice images are put together using somewhat ad hoc weighting functions. In this work, we demonstrate a regridding method based on [24] that directly utilizes the depth information into the tomographic reconstruction, allowing a concomitant reduction of the number of projections needed for appropriate angular sampling. Compared to earlier work [21-23], it performs the reconstruction in a single step that includes both rotation and fusion, based on a well-defined interpolation kernel whose effect is removed at the last step through deconvolution. Our method offers a general, non-iterative, and numerically efficient solution to the tomographic reconstruction problem, applicable to any imaging scheme that provides depth-resolved projections. We term it the Gridrec multi-slice method (Gridrec-MS).

Illustrations of conventional tomography with no depth information and multi-slice tomography are given in Figs. 1(a) and 1(b), respectively. For conventional tomography in a parallel beam geometry, the problem can be simplified to mutually independent reconstructions of $2 \mathrm{D}$ disks perpendicular to the rotation axis. Hence, from here on we focus on the reconstruction of these 2D disks from $1 \mathrm{D}$ projections. A 2D disk of an object is denoted as $f\left(r_{x}, r_{y}\right)$ on the Cartesian grid and its Fourier transform as $F\left(u_{x}, u_{y}\right)$. For a given rotation of the object, $\theta$, the object can be expressed as $f(r, z)$ where $z$ is the beam propagation direction, $r=\left(r_{x} \cos \theta+r_{y} \sin \theta\right)$ and its Fourier transform $F(u, w)$. These real-space and Fourier-space coordinates are illustrated in Fig. 1. In conventional tomography, a projection measured at $\theta$ is expressed as $p_{\theta}(r)=\int_{-\infty}^{\infty} f(r, z) \mathrm{d} z$. Using the Fourier-slice theorem [25], projections at multiple angles can yield a reconstruction, as illustrated in Fig. 1(a) by 


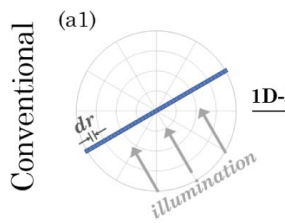

$$
\text { (a2) }
$$

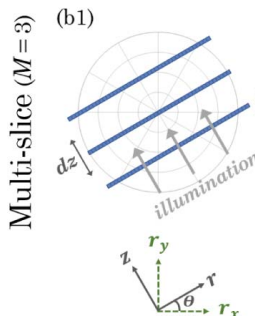

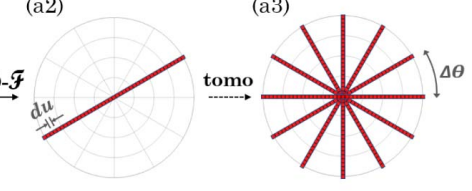

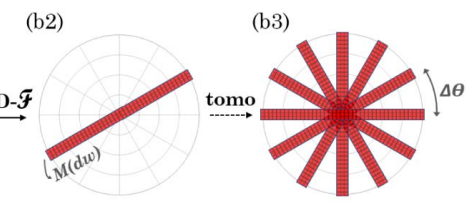

Fig. 1. Illustration of the Fourier space coverage for (a) conventional projection tomography and (b) multi-slice tomography with $M=3$. A depth-resolved projection has a dimension of $M$ by $T$ and, with the multi-slice method, its 2D Fourier transform [27] is thickened by $M$ compared to that of the conventional projection. As a result, more of the Fourier space is filled with the data for the same $\Delta \theta$.

$$
f\left(r_{x}, r_{y}\right)=\int_{0}^{\pi}\left[\int_{-\infty}^{\infty} P_{\theta}(u) R(u) \exp (i 2 \pi u r) \mathrm{d} u\right] \mathrm{d} \theta,
$$

where $P_{\theta}(u)$ is the Fourier transform of $p_{\theta}(r)$ and $R(u)$ is the ramp filter $|u|$. For an imaging system of transverse resolution or pixel size of $\delta r$ and an object thickness of $T$, the required number of projections to achieve $3 \mathrm{D}$ isotropic resolution $\delta r$ is given by

$$
N_{\theta}=180^{\circ} / \Delta \theta=\alpha T /(d r),
$$

where $\Delta \theta$ is the angular spacing and the coefficient $\alpha$ is $\pi / 2$ based on the Crowther's criterion [25,26].

With specific experimental techniques, image processing, or physical modelling, the depth information can in some cases be extracted from a projection of the object to give virtual slices or virtual sections of the object, here referred to as a depthresolved projection, shown in Fig. 1(b1). Multi-slice methods exist in the optical $[8,14,21-23]$ and X-ray regimes $[9,10]$ that obtain such depth sectioning. Such a projection has a dimension of $M$ by $T$, where $M$ is the number of sections and $T$ the object diameter, and is expressed as

$$
\hat{p}_{\theta}(r, m)=\int_{z_{m^{\prime}}}^{z_{m^{\prime \prime}}} f(r, z) \mathrm{d} z, \quad 1 \leq m \leq M,
$$

where $\left(z_{m^{\prime}}, z_{m^{\prime \prime}}\right)$ is the range for section $m$ in the $z$ direction. The 2D Fourier transform of $\hat{p}_{\theta}(r, m)$ is calculated by the fast Fourier transform algorithm [28] and denoted as $\hat{P}_{\theta}(u, w)$, which is defined on the rotated Cartesian coordinates $(u, w)$ or, in the case where $M=1$, the polar coordinates. The advantage of incorporating depth information in the tomographic reconstruction is illustrated in Fig. 1. The multi-slice projections are thickened by $M$ in Fourier space [15], as illustrated in Fig. 1(b2), compared to a conventional projection in Fig. 1(a2). Therefore more of the Fourier space is covered for a given angular sampling $\Delta \theta$, as shown in Figs. 1(a3) and 1 (b3). This also means that with the multi-slice method, the number of projections required to cover the Fourier space is in principle reduced to [15]

$$
\hat{N}_{\theta}=N_{\theta} / M \text {, }
$$

where $N_{\theta}$ is defined in Eq. (2) and $\hat{N}_{\theta}$ is the number of projections needed if using the multi-slice method with $M$ sections. Equivalently this can be thought as $\Delta \hat{\theta}=M \Delta \theta$, which indicates that the Crowther criterion is reduced by a factor equal to the number of sections [15].

Here we introduce a modified regridding method, GridrecMS, that allows for direct tomographic reconstruction using the multi-slice depth information. An overview of the method is given in Fig. 2. A regridding technique [24] maps data to Cartesian coordinates after convolution with a kernel whose contribution is later removed in real space, i.e., after the $2 \mathrm{D}$ inverse Fourier transform. Interpolation in 2D Fourier space is the critical step of Direct Fourier methods as local interpolation inaccuracies in Fourier space propagate to all pixels in the real-image space. With a convolutional kernel $C(u, w)$, the tomographic reconstruction can be expressed as

$$
\begin{aligned}
f\left(r_{x}, r_{y}\right)= & \left\{\int_{-\infty}^{\infty} \int_{-\infty}^{\infty}\left[\int_{0}^{\pi} \hat{P}_{\theta}(u, w) * C(u, w) \mathrm{d} \theta\right] \hat{R}\left(u_{x}, u_{y}\right)\right. \\
& \left.\times \exp \left[i 2 \pi\left(r_{x} u_{x}+r_{y} u_{y}\right)\right] \mathrm{d} u_{x} \mathrm{~d} u_{y}\right\} c^{-1}\left(r_{x}, r_{y}\right),
\end{aligned}
$$

where $*$ denotes the convolution operation, $\hat{R}\left(u_{x}, u_{y}\right)$ is a $2 \mathrm{D}$ filter which serves a similar normalization purpose as the ramp filter for conventional tomography, and $c\left(r_{x}, r_{y}\right)$, the inverse Fourier transform of $C(u, w)$, effectively compensates the effect of the kernel. Equation (5) can also be written as

$$
\begin{aligned}
f\left(r_{x}, r_{y}\right)= & \left\{\int_{-\infty}^{\infty} \int_{-\infty}^{\infty} Q\left(u_{x}, u_{y}\right) \hat{R}\left(u_{x}, u_{y}\right)\right. \\
& \left.\times \exp \left[i 2 \pi\left(r_{x} u_{x}+r_{y} u_{y}\right)\right] \mathrm{d} u_{x} \mathrm{~d} u_{y}\right\} c^{-1}\left(r_{x}, r_{y}\right),
\end{aligned}
$$

where the Fourier map

$$
Q\left(u_{x}, u_{y}\right)=\int_{0}^{\pi} \hat{P}_{\theta}(u, w) * C(u, w) \mathrm{d} \theta=\int_{0}^{\pi} Q_{\theta}\left(u_{x}, u_{y}\right) \mathrm{d} \theta
$$

is the result of interpolating multi-slice projections, $\hat{P}_{\theta}(u, w)$, onto the Cartesian grid $\left(u_{x}, u_{y}\right)$ through $C(u, w)$. The choice of the interpolation kernel is crucial as functions with a compact support both in real and Fourier space provide higher accuracy [29] and high computational efficiency. Here we use a separable form of the kernel, which gives $C(u, w)=C(u) C(w)$, where $C(u)$ is formed by prolate spheroidal wave functions (PSWFs) of zeroth order [30]. For efficient computation and storage, expansions of PSWFs [31] in terms of Legendre polynomials are used here [24], giving

$$
C(u)=\sum_{k=0}^{20} \beta_{k} P_{k}(u),
$$

where $\beta_{k}$ is the coefficient for the $k$-th degree Legendre polynomial $P_{k}$ and $k$ covers the expansion to a sufficient degree. Figure 2(a) illustrates the mapping of one pixel of $\hat{P}_{\theta}(u, w)$ onto Cartesian coordinate $\left(u_{x}, u_{y}\right)$. The non-zero components 


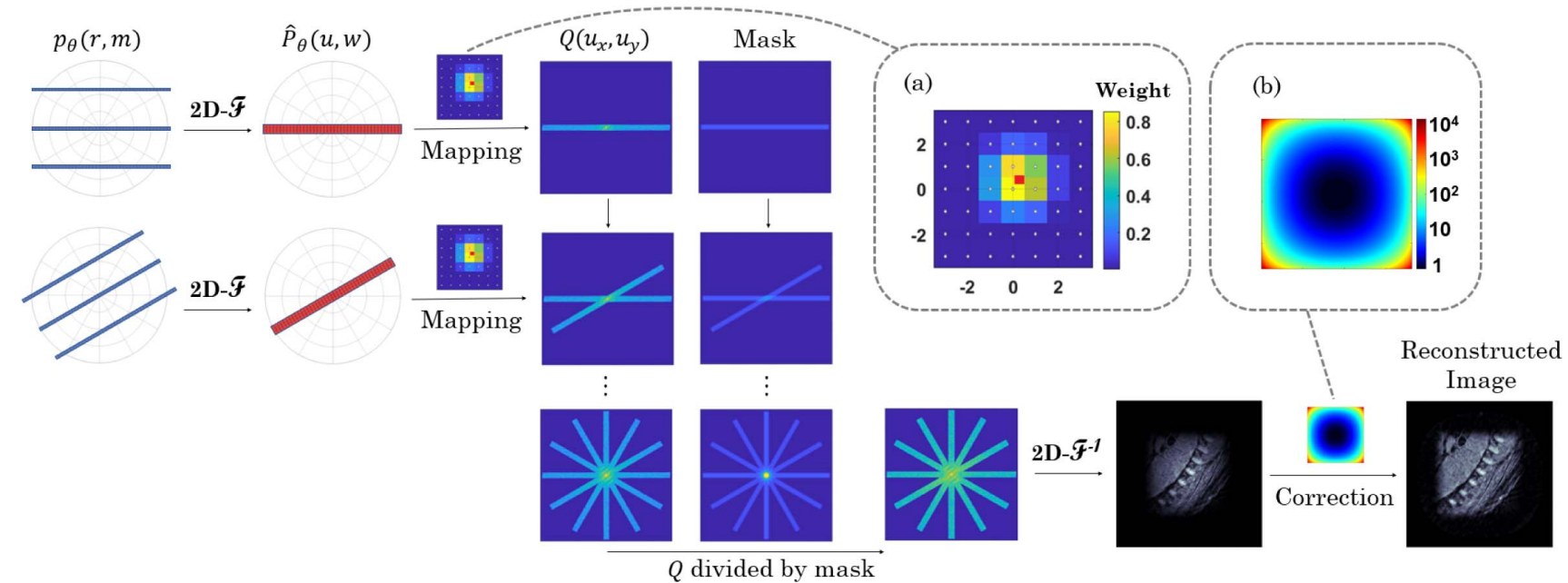

Fig. 2. Overview of Gridrec-MS, related to Eq. (5). 2D Fourier transform of the projections were mapped to Cartesian coordinates $\left(u_{x}, u_{y}\right)$ through (a) an interpolation matrix $C(u, w)$. A normalization mask is created based on the non-zero elements of $C(u, w)$ at all points of $\hat{P}_{\theta}(u, w)$ and for all angles. After interpolation, the resulting Fourier map $Q\left(u_{x}, u_{y}\right)$ is divided by this mask to ensure equal contribution from all projections. (b) A correction function in real space, $c\left(r_{x}, r_{y}\right)$, is used to remove the contribution of the interpolation kernel in (a).

of this interpolation map are added as ones to a mask that will later be used for normalization. For every point of $\hat{P}_{\theta}(u, w)$ such interpolation is performed. The process is repeated for all angles, resulting in a Fourier space that is covered by all multislice projections as well as a mask for normalization. The Fourier map $Q\left(u_{x}, u_{y}\right)$ is then divided by the mask, expressed as a multiplication by $\hat{R}\left(u_{x}, u_{y}\right)$ in Eq. (6), to normalize the contribution from each projection. Subsequently, a 2D inverse
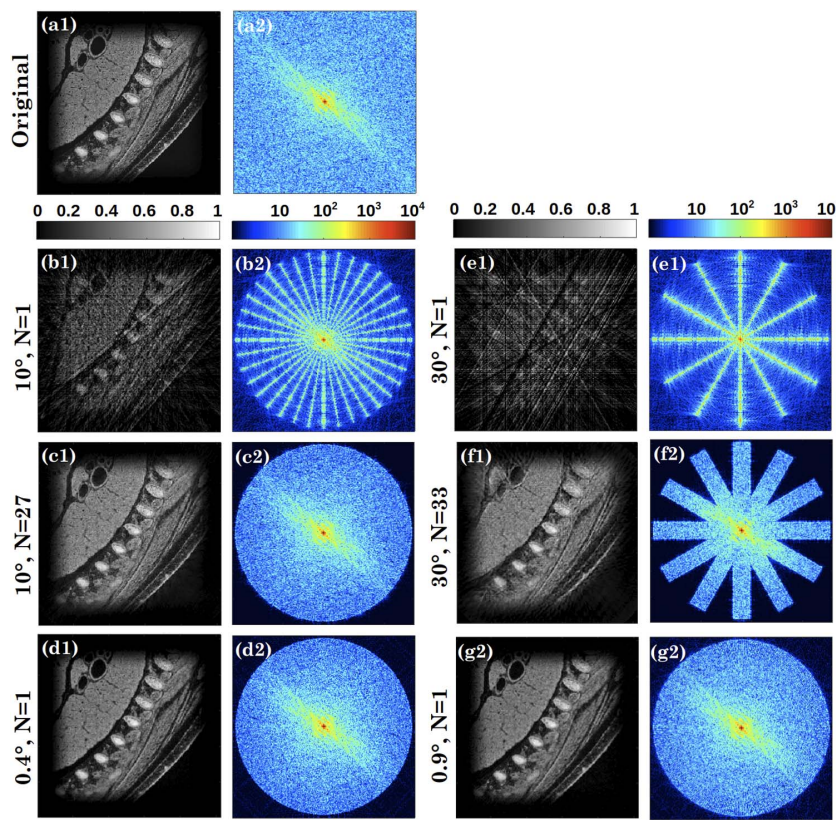

Fig. 3. Multi-slice tomography using Gridrec-MS. (a1) Original object and (a2) its Fourier map. Reconstruction from projections simulated at $\theta=10^{\circ}$ with (b) conventional projections and with (c) multi-slice projections with $N=27$. (d) Conventional projection tomography using fine sampling at $\theta=0.4^{\circ}$. Reconstructions for (e) $-(\mathrm{f}) \theta=30^{\circ}$ and (g) $\theta=0.9^{\circ}$.
Fourier transform is performed and a correction matrix in real space, $c\left(r_{x}, r_{y}\right)$, is applied to remove the effect of the interpolation kernel. The intensity of the correction function is shown in Fig. 2(b). For simplicity, these equations are given in continuous space while a discretized version was implemented.

The effectiveness of Gridrec-MS is demonstrated in Fig. 3 for different angular samplings. In order to have a numerical phantom with a representative distribution of spatial frequencies, a mouse histology image [32] was used as the object, as shown in Fig. 3(a). To achieve single-pixel resolution, the angular sampling for an object size of $256 \times 256$ pixels is $\Delta \theta=$ $0.45^{\circ}$ according to Eq. (2). Conventional projection tomography with $\Delta \theta=10^{\circ}$ was simulated and reconstructed with a ramp filter using Gridrec, as shown in Fig. 3(b). With the same angular sampling, Gridrec-MS was used for tomographic reconstruction of projections with $M=27$ sections. Each depth-resolved projection was generated following Eq. (3). The filter $\hat{R}\left(u_{x}, u_{y}\right)$ here equals the division by a normalization mask. The mask is defined by the non-zero components from each projection, as described previously, and schematically shown in Fig. 2. Using Gridrec-MS in this case provides a complete coverage of the Fourier space and a significantly improved image quality, as shown in Fig. 3(c). The obvious improvement from Figs. 3(b1) to 3(c1) demonstrates the relaxation of the Crowther criterion [15] and it validates Gridrec-MS. The insufficient angular sampling results in irreparable loss of information in Fourier domain, illustrated by the angular gaps in Fig. 3(b2), also reflecting the fine features missing in Fig. 3(b1). For comparison, Fig. 3(d) shows the conventional projection tomography with an angular spacing of $\Delta \theta=10^{\circ} / 27 \approx 0.4^{\circ}$, which shows comparable quality with Figs. 3(a) and 3(c). The reconstruction quality is given in Fig. 4, evaluated quantitatively based on Fourier shell correlation (FSC) [33] curves calculated between the reconstruction and the original object. In Fig. 4(a), both results from Figs. 3(c) and 3(d) give single-pixel resolution using the 1-bit threshold criterion. The reconstruction quality for the multi-slice method 
(a)
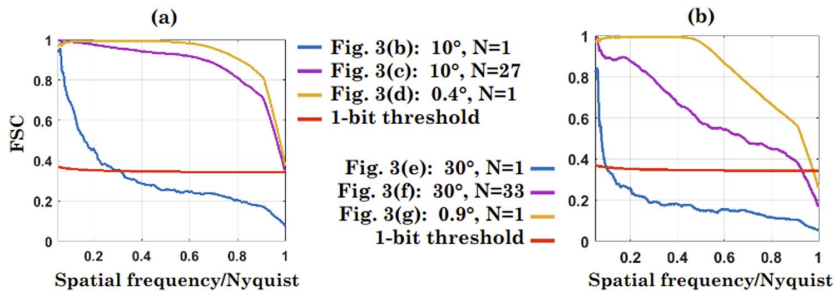

Fig. 4. FSC curves of the reconstructions in Fig. 3. The 1-bit threshold is shown in red. In both cases, multi-slice reconstructions (purple) show great improvement over the conventional method (blue) and slightly worse results compared to single-slice with fine angular sampling (yellow)

is, however, slightly lower as shown by the FSC curve in purple compared to the yellow for the conventional method.

Figures 3(e) and 3(f) show the conventional reconstruction with an angular sampling of $\Delta \theta=30^{\circ}$ and the multi-slice reconstruction with the same angular sampling but with $M=33$. Neither cases have adequate angular sampling, but it is clear that multi-slice tomography reconstruction leverages the depth information to fill more of the Fourier space and give recognizable features, shown by the dramatic difference between Figs. 3(e) and 3(f). As comparison, a conventional tomogram using data with $\Delta \theta=30^{\circ} / 33=0.9^{\circ}$ is given in Fig. 3(g). The imperfection in Fig. 3(f1) as well as the overall lower correlation of the FSC curve in purple compared to the yellow one in Fig. 4(b) corresponds to the missing data shown in the Fourier space in Fig. 3(f2).

We have demonstrated Gridrec-MS for direct multi-slice tomographic reconstruction, i.e., using depth-resolved projections. Reconstructions using projections with $M$ sections are comparable to those with conventional single-slice fine sampling, demonstrating that the Crowther criterion [15] can be relaxed by a factor of $M$ compared to the regular sampling requirements. In other words, the number of sections, $M$, influences the angular sampling. We have also shown that even with sparse angular sampling, the reconstruction can still benefit significantly from the depth information. In practice the multislice sectioning, provided by the imaging method upstream to Gridrec-MS, may not be perfect. If such imperfections are not considered, they can affect the quality of the reconstruction. For example, some methods show a dependence of the sectioning efficacy with respect to the lateral spatial frequency, in which low-spatial-frequency features leak to neighboring object sections, leading to a transfer function with a support in the shape of a bowtie $[4,10]$. The method presented here can be adapted for this situation by a modification of $\hat{R}\left(u_{x}, u_{y}\right)$, i.e., changing the Fourier mask. In general, it can be advantageous to combine techniques that model the imaging system to extend the DOF or obtain sectioning with tomographic methods. For imaging systems for which the depth information can be recovered, we expect that Gridrec-MS can provide a robust and general reconstruction framework that is readily applicable for any contrast mechanism, including phase and absorption contrast, as well as any probing wave, including electrons, $\mathrm{X}$-rays, and optical photons. The Gridrec-MS code can be made available upon reasonable request.
Funding. Swiss National Science Foundation (200020 169623, 200021_152554); U.S. Department of Energy (DOE) (DE-SC0012704).

\section{ACKNOWLEDGMENT}

We thank Alain Studer at Paul Scherrer Institut for the fruitful discussions.

\section{REFERENCES}

1. M. Levoy, R. Ng, A. Adams, M. Footer, and M. Horowitz, ACM Trans. Graph. 25, 924 (2006).

2. E. R. Dowski and W. T. Cathey, Appl. Opt. 34, 1859 (1995).

3. R. H. Webb, Rep. Prog. Phys. 59, 427 (1996).

4. M. Neil, R. Juškaitis, and T. Wilson, Opt. Lett. 22, 1905 (1997).

5. J.-A. Conchello and J. W. Lichtman, Nat. Methods 2, 920 (2005).

6. S. A. Shroff, J. R. Fienup, and D. R. Williams, J. Opt. Soc. Am. A 26, 413 (2009).

7. R. Hovden, H. L. Xin, and D. A. Muller, Microsc. Microanal. 17, 75 (2011).

8. A. M. Maiden, M. J. Humphry, and J. M. Rodenburg, J. Opt. Soc. Am. A 29, 1606 (2012).

9. A. Suzuki, S. Furutaku, K. Shimomura, K. Yamauchi, Y. Kohmura, T. Ishikawa, and Y. Takahashi, Phys. Rev. Lett. 112, 053903 (2014).

10. E. H. R. Tsai, I. Usov, A. Diaz, A. Menzel, and M. Guizar-Sicairos, Opt. Express 24, 29089 (2016).

11. K. Shimomura, A. Suzuki, M. Hirose, and Y. Takahashi, Phys. Rev. B 91, 214114 (2015).

12. K. Shimomura, M. Hirose, and Y. Takahashi, Acta Crystallogr. Sec. A 74, 66 (2018)

13. E. H. R. Tsai, M. Odstrcil, I. Usov, M. Holler, A. Diaz, J. Bosgra, A. Menzel, and M. Guizar-Sicairos, in Computational Optical Sensing and Imaging (Optical Society of America, 2017), paper CW3B.2.

14. P. Li and A. Maiden, Sci. Rep. 8, 2049 (2018).

15. C. Jacobsen, Opt. Lett. 43, 4811 (2018).

16. K. Shimomura, M. Hirose, T. Higashino, and Y. Takahashi, Opt. Express 26, 31199 (2018).

17. A. C. Kak and M. Slaney, Principles of Computerized Tomographic Imaging (IEEE, 1988).

18. R. Gordon, R. Bender, and G. T. Herman, J. Theor. Biol. 29, 471 (1970).

19. B. A. Dowd, G. H. Campbell, R. B. Marr, V. V. Nagarkar, S. V. Tipnis, L. Axe, and D. P. Siddons, Proc. SPIE 3772, 224 (1999).

20. M. A. Gilles, Y. S. G. Nashed, M. Du, C. Jacobsen, and S. M. Wild, Optica 5, 1078 (2018).

21. P. J. Shaw, D. A. Agard, Y. Hiraoka, and J. W. Sedat, Biophys. J. 55 101 (1989).

22. J. Swoger, J. Huisken, and E. H. Stelzer, Opt. Lett. 28, 1654 (2003).

23. J. Swoger, P. Verveer, K. Greger, J. Huisken, and E. H. Stelzer, Opt. Express 15, 8029 (2007).

24. F. Marone and M. Stampanoni, J. Synchrotron Radiat. 19, 1029 (2012).

25. K. C. Avinash and M. Slaney, Principles of Computerized Tomographic Imaging (Society of Industrial and Applied Mathematics, 2001).

26. R. A. Crowther, D. DeRosier, and A. Klug, Proc. R. Soc. London Ser. A 317, 319 (1970).

27. J. W. Cooley and J. W. Tukey, Math. Comput. 19, 297 (1965).

28. M. Frigo and S. G. Johnson, Proc. IEEE 93, 216 (2005), Special issue on Program Generation, Optimization, and Platform Adaptation.

29. J. O'Sullivan, IEEE Trans. Med. Imaging 4, 200 (1985).

30. D. Slepian and H. O. Pollak, Bell Syst. Tech. J. 40, 43 (1961).

31. H. Xiao, V. Rokhlin, and N. Yarvin, Inverse Prob. 17, 805 (2001).

32. http://brainmaps.org (2015).

33. M. van Heel and M. Schatz, J. Struct. Biol. 151, 250 (2005). 